\title{
Dynamics Analysis and Optimality in Selective Harvesting Predator-Prey Model With Modified Leslie-Gower and Holling-Type $I I$
}

https://doi.org/10.1515/msds-2019-0001

Received April 2, 2018; accepted February 25, 2019

\begin{abstract}
In this work, we consider the optimal harvesting and stability problems of a prey-predator model with modified Leslie-Gower and Holling-type II functional response. The model is governed by a system of three differential equations which describe the interactions between prey, predator and harvesting effort. Boundedness and existence of solutions for this system are showed. The existence and local stability of the possible steady states are analyzed and the conditions of global stability of the interior equilibrium are established by using the Lyapunov function, we prove also the occurrence of Hopf bifurcation at this point. By using the Pontryagin's maximal principle, we formulate and we solve the problem of the optimal harvest policy. In the end, some numerical simulations are given to support our theoretical results.
\end{abstract}

Keywords: Predator-prey model, ordinary differential equations, local and global stability, bifurcation, optimal harvesting policy

\section{Introduction and Mathematical model}

In the last recent, the mathematical modeling of prey-predator models since Lotka (1925) and Volterra (1927) is a very interesting area of research for many ecologists, mathematicians and economists. The problem of optimal harvesting in predator-prey systems is a dominant theme in ecology and bio-economics in due to its importance. Many authors have fired their models in this theme of Clark [1] who proved the optimal equilibrium policy for joint harvesting of two independent species and he suppose that each population follows a logistic growth law in the absence of harvesting and its harvest rate is proportional to both its stock level and harvesting effort. This analysis has been studied and analyzed by several authors Braverman and Mamdani [4], Fan and Wang [2], Qiu et al [3].

There are also numerous works on the effect of harvesting on predator-prey interaction, Kar [8], Brauer and Soudack [15], Pal et al.[9], Kalyan [11], Agarwal [12], Xiao and Ruan [13] and Myerscough et al. [14].

Many researchers concentrate their research work in the field of optimal management of renewable resources. Costa et al. [16] considered Lotka-Volterra and Leslie-Gower predator-prey model and proved the global stability of a desired equilibrium population. Rebaza [18] studied the boundedness of solutions, existence of bionomic equilibria, positivity of equilibrium states, local and global stability of equilibrium points and peri-

W. Abid: Laboratoire d'ingenierie Mathématique de l'ecole polytechnique de Tunis, E-mail: abidwalid007@yahoo.fr ^Corresponding Author: R. Yafia: Ibn Tofail Universit, Faculty of Sciences, Department of Mathematics, Campus Universitaire, BP 133, Kénitra, Morocco, E-mail: yafia1@yahoo.fr

M. A. Aziz-Alaoui: LMAH, FR-CNRS-3335, Université du Havre Normandie, 25 Rue Ph. Lebon, BP:540, 76058 Le Havre Cedex, (Normandie) France, E-mail: aziz.alaoui@univ-lehavre.fr

Ahmed Aghriche: Ibn Zohr University, CST Campus Universitaire Ait Melloul Agadir, Morocco, E-mail: aghriche87@gmail.com 
odic solutions of a predator-prey model with harvesting. Das et al. [23] introduced prey-predator harvesting model with toxicity and discussed optimal harvesting policy using Pontryagin's maximal principle. Fister and Lenhart [30] studied a predator-prey model, where the predator is represented by the Lotka-Volterra ordinary differential equation. Zhang and chen [31] considered the stage structured predator prey model and optimal harvesting policy. Always in this topic, Beddington and May [25], Liu [24], Li and Wang [26] have studied the optimal harvesting strategy of stochastic population systems (species affected by environmental natural factors) and some other authors have discussed and analyzed a prey-predator model with harvesting.

The exploitation of biological resources and the harvest of population species are commonly experienced in fishery, forestry and wildlife management. In the natural world and among the serious and immediate global problem for future fisheries management and sustainable development of ecosystem, is over exploitation of fisheries, different species of fishes are decreased due to enhancement of fishing power, high growth rate of world population and lack of knowledge of the characteristics of exploited species. Therefore several authors have studied the optimal harvest policy in fishery.

Palma [27] found the optimal harvest policy in an open access fishery in which both prey and predator populations are exposed to non selective harvesting and the growth rate of prey species is affected by Allee-effect. Chen and Hsui [28] investigated the fishery policy when considering the future opportunity of harvesting. Dubey and Chandra [29] investigated the dynamics of a fisher resource system in an aquatic environment in two zones harvest in reserve area.

In Roy and Roy [42], the authors formulated a mathematical model describing the interactions between three species: super predator-predator-prey with different types of functional responses. The conditions of local and global stabilities of the interior equilibrium are given. In Roy and Roy [43], a model with four compartments of prey (refuge region-predatory part) and predator (super predator-predator) and only predator population is harvested. The obtained model is studied in terms of stability and Hopf bifurcation. To optimize the utilization of the resource, the authors considered the fishing effort as a control to harvest predator population. On the other hand, in Roy et al. [45] a mathematical model is introduced to investigate the effect on prey of two predators (predator-generalist predator) and the the effect of the harvesting effort for the generalist predator. The conditions of stabilities and Hopf bifurcation are established. In Roy et al. [44], the authors studied the effect of harvesting on prey and the affect of time delay on a formulated Holling-Tanner prey-predator model with Beddington-DeAngelis functional response. They studied the stability of the interior equilibrium and the existence of small amplitude periodic solutions and their stability via the Hopf bifurcation Theorem. Motivated by the above works, we consider the following predator-prey model which incorporates the Holling type II and a modified Leslie-Gower functional response (see Aziz Alaoui et al. [5]) and takes into account the harvesting in prey population and integrates the equation of variation of the effort of harvesting:

$$
\left\{\begin{array}{l}
\frac{d u}{d t}=\left(a_{1}-b_{1} u-\frac{c_{1} v}{u+k_{1}}\right) u-h(t), \\
\frac{d v}{d t}=\left(a_{2}-\frac{c_{2} v}{u+k_{2}}\right) v,
\end{array}\right.
$$

the variable $u$ is the density of prey population and $v$ is the density of predator population at any instant of time $t$ subject to the non-negative initial condition $u(0)>0$ and $v(0)>0$, the term $\frac{c_{2} v}{u+k_{2}}$ denotes the functional response of the predator, which is known as Holling type II response function, $h(t)>0$ is the rate of prey harvesting.

According the catch per unit effort hypothesis [41], the functional form of harvest is generally considered to define the assumption that the catch per unit effort is proportional to the stock level. The harvest function depends on several factors, revenue, market demand and harvest cost. Therefore, we consider that the harvest effort is a variable from the real point of view, which takes the following form:

$$
h(t)=m q u w,
$$

where $w$ is the effort used to harvest the population, $m(0<m<1)$ is the fraction of the stock available for harvesting and $q$ is the catchability coefficient. 
We suppose that fishery effort itself is a dynamic variable that satisfies

$$
\frac{d w}{d t}=\lambda(p m q u-c) w
$$

where $p$ is the price per unit biomass of landed fish, $c$ is the constant fishing cost per unit effort and $\lambda$ is stiffness parameter.

In this paper, we have considered the following ordinary differential equations:

$$
\left\{\begin{array}{l}
\frac{\partial u}{\partial t}=\left(a_{1}-b_{1} u-\frac{c_{1} v}{u+k_{1}}\right) u-m q u w \\
\frac{\partial v}{\partial t}=\left(a_{2}-\frac{c_{2} v}{u+k_{2}}\right) v \\
\frac{\partial w}{\partial t}=\lambda(p m q u-c) w
\end{array}\right.
$$

with initial conditions $u(0)=u_{0} \geq 0, v(0)=v_{0} \geq 0, w(0)=w_{0} \geq 0$.

Our goal, in this paper is to study the dynamics of model presented in paper of Aziz Alaoui and Daher [5] but with presence of harvesting and adding a dynamic variable is fishery effort.

In the absence of harvesting, the first model proposed in this optic is given by Aziz Aloui et al.[5] as follows:

$$
\left\{\begin{array}{l}
\frac{d u}{d t}=\left(a_{1}-b_{1} u-\frac{c_{1} v}{u+k_{1}}\right) u, \\
\frac{d v}{d t}=\left(a_{2}-\frac{c_{2} v}{u+k_{2}}\right) v,
\end{array}\right.
$$

with initial conditions $u(0)>0$ and $v(0)>0, u$ and $v$ represent population densities at time $t$. $c_{1}, a_{1}, b_{1}, k_{1}, c_{2}, a_{2}$ and $k_{2}$ are model parameters assuming only positive values. $a_{1}$ is the growth rate of preys $u . a_{2}$ describes the growth rate of predators $v . b_{1}$ measures the strength of competition among individuals of species $u . c_{1}$ is the maximum value of the per capita reduction of $u$ due to $v . c_{2}$ has a similar meaning to $c_{1}$. $k_{1}$ measures the extent to which environment provides protection to prey $u$. $k_{2}$ has a similar meaning to $k_{1}$ relatively to the predator $v$.

In $[5,6]$, the authors investigated this model in terms of boundedness of solutions, existence of a positively invariant and attracting set, stability analysis of the coexisting interior equilibrium, permanence and extinction, existence and uniqueness of limit cycle. The notion of global stability, existence and uniqueness of limit cycle and Hopf bifurcation are showed by Hsu et al. [32, 33, 39] and Chakraborty et al. [40]. In [35, 36], Yafia et al. studied a delayed version of this model, they showed the existence of periodic solutions and their stability. The global stability and persistence are proved in $[37,38]$ by using lyapunov function.

The organization of this paper is as follows. In section 2, we prove the existence of the equilibrium points and the local stability of the trivial steady state and the boundedness of solutions. Section 3 is devoted to the local and global stability of the nontrivial steady state. In section 4, we establish the conditions of the occurrence of Hopf bifurcation. In Section 5, we solve the problem of determining the optimal harvest policy by using Pontryagin's maximal principle. Numerical simulations are performed to illustrate the effectiveness of our results in section 6 .

\section{Preliminaries}

\subsection{Boundedness, boundary equilibria}

In this subsection, we recall some results on model (1.4) such as boundedness, existence of boundary equilibria and their stability.

Theorem 2.1. Let $\Theta$ be the set defined by

$$
\Theta=\left\{(u, v, w) \in \mathbb{R}_{+}^{3}, 0 \leq u \leq \frac{a_{1}}{b_{1}}, 0 \leq u+v+\frac{w}{\lambda p} \leq \frac{L}{c \lambda}\right\}
$$


where $L=\frac{\left(a_{1}+c \lambda\right)^{2}}{4 b_{1}}+\frac{\left(a_{2}+c \lambda\right)^{2}\left(a_{1}+k_{2} b_{1}\right)}{4 b_{1} c_{2}}$

i) $\Theta$ is positively invariant region.

ii) All solutions of (1.4) initiating in $\Theta$ are ultimately bounded with respect to $\mathbb{R}_{+}^{3}$ and eventually enter the attracting set $\Theta$.

Proof. Since $(u(t), v(t), w(t))$ is a positive solution of (1.4) initiating from the positive initial condition $(u(0), v(0), w(0))$, we have

$$
\left\{\begin{array}{l}
\frac{\partial u(t)}{\partial t} \leq\left(a_{1}-b_{1} u(t)\right) u(t), \\
u(0)=u_{0} \geq 0 .
\end{array}\right.
$$

From Lemma (3.2) of chen [17], we deduce that

$$
\limsup _{t \rightarrow+\infty} u(t) \leq \frac{a_{1}}{b_{1}} .
$$

Let the function $\sigma(t)=u(t)+v(t)+\frac{w(t)}{\lambda p}$, by time derivative we have

$$
\begin{aligned}
\frac{d \sigma(t)}{d t}+c \lambda \sigma(t) & =\frac{u(t)}{d t}+\frac{v(t)}{d t}+\frac{1}{\lambda p} \frac{w(t)}{d t}+c \lambda\left(u(t)+v(t)+\frac{w(t)}{\lambda p}\right) \\
& =\left(a_{1}-b_{1} u-\frac{c_{1} v}{u+k_{1}}\right) u-m q u w+\left(a_{2}-\frac{c_{2} v}{u+k_{2}}\right) v+\frac{\lambda}{\lambda p}(p m q u-c) w+c \lambda\left(u(t)+v(t)+\frac{w(t)}{\lambda p}\right) \\
& =\left(a_{1}-b_{1} u-\frac{c_{1} v}{u+k_{1}}\right) u+\left(a_{2}-\frac{c_{2} v}{u+k_{2}}\right) v+c \lambda(u(t)+v(t)), \\
& =\left(a_{1}+c \lambda-b_{1} u-\frac{c_{1} v}{u+k_{1}}\right) u+\left(a_{2}+c \lambda-\frac{c_{2} v}{u+k_{2}}\right) v
\end{aligned}
$$

As $u$ and $v$ are positives and from (2.2), we get

$$
\begin{aligned}
\frac{d \sigma(t)}{d t}+c \lambda \sigma(t) & \leq \frac{\left(a_{1}+c \lambda\right)^{2}}{4 b_{1}}+\left(a_{2}+c \lambda-\frac{c_{2} v}{\frac{a_{1}}{b_{1}}+k_{2}}\right) v \\
& \leq \frac{\left(a_{1}+c \lambda\right)^{2}}{4 b_{1}}+\frac{\left(a_{2}+c \lambda\right)^{2}\left(a_{1}+k_{2} b_{1}\right)}{4 b_{1} c_{2}}, \\
& \leq L,
\end{aligned}
$$

where $L=\frac{\left(a_{1}+c \lambda\right)^{2}}{4 b_{1}}+\frac{\left(a_{2}+c \lambda\right)^{2}\left(a_{1}+k_{2} b_{1}\right)}{4 b_{1} c_{2}}$.

From [46], we obtain

$$
0 \leq \sigma(t) \leq \frac{L}{c \lambda}+\frac{\sigma(0)}{e^{c \lambda t}}
$$

and for $t \longrightarrow+\infty, 0 \leq \sigma(t) \leq \frac{L}{c \lambda}$.

Thus, all solutions of the system (1.4) enter into the region $\left\{(u, v, w) \in \mathbb{R}_{+}^{3}, 0 \leq u \leq \frac{a_{1}}{b_{1}}+\varepsilon, 0 \leq u+v+\frac{w}{\lambda p} \leq \frac{L}{c \lambda}+\varepsilon\right\}$. This complete the proof of the theorem.

Lemma 2.1. The possible steady states of system (1.4) are $E_{0}=(0,0,0), E_{1}=\left(\frac{a_{1}}{b_{1}}, 0,0\right), E_{2}=\left(0, \frac{a_{2} k_{2}}{c_{2}}, 0\right)$, $E_{3}=\left(\widehat{u_{3}}, \widehat{v_{3}}, 0\right)$, exists if and only if

$$
\begin{gathered}
a_{1}^{2} c_{2}^{2}+2 a_{1} b_{1} c_{2}^{2} k_{1}+a_{2}^{2} c_{1}^{2}+2 a_{2} b_{1} c_{1} c_{2} k_{1}+b_{1}^{2} c_{2}^{2} k_{1}^{2}>2 a_{1} a_{2} c_{1} c_{2}+4 k_{2} a_{2} b_{1} c_{1} c_{2}, \\
a_{1} a_{2} c_{2}+2 k_{2} a_{2} b_{1} c_{2}>a_{2}^{2} c_{1}+a_{2} b_{1} c_{2} k_{1}, \\
a_{2} \sqrt{s}-a_{2}^{2} c_{1}+a_{1} a_{2} c_{2}-a_{2} b_{1} c_{2}\left(k_{1}-2 k_{2}\right)>2 k_{2} a_{2} b_{1} c_{2},
\end{gathered}
$$

with

$$
s=a_{1}^{2} c_{2}^{2}-2 a_{1} a_{2} c_{1} c_{2}+2 a_{1} b_{1} c_{2}^{2} k_{1}+a_{2}^{2} c_{1}^{2}+2 a_{2} b_{1} c_{1} c_{2} k_{1}-4 k_{2} a_{2} b_{1} c_{1} c_{2}+b_{1}^{2} c_{2}^{2} k_{1}^{2},
$$




$$
\begin{gathered}
\widehat{u_{3}}=\frac{\left(a_{2} \sqrt{s}\right)-a_{2}^{2} c_{1}+a_{1} a_{2} c_{2}-a_{2} b_{1} c_{2}\left(k_{1}-2 k_{2}\right)}{2 a_{2} b_{1} c_{2}}-k_{2}, \\
\widehat{v_{3}}=\frac{\left(a_{2} \sqrt{s}\right)-a_{2}^{2} c_{1}+a_{1} a_{2} c_{2}-a_{2} b_{1} c_{2}\left(k_{1}-2 k_{2}\right)}{2 b_{1} c_{2}^{2}},
\end{gathered}
$$

$E_{4}=\left(\widehat{u_{4}}, 0, \widehat{w_{4}}\right)$ exists if and only if $a_{1} p m q>b_{1} c$, with $\widehat{u_{4}}=\frac{c}{p m q}$ and $\widehat{w_{4}}=\frac{a_{1} p m q-b_{1} c}{p m^{2} q^{2}}$.

The jacobian matrix associated with the system (1.4),

$$
J(u, v, w)=\left(\begin{array}{ccc}
a_{1}-2 b_{1} u-\frac{c_{1} k_{1} v}{\left(u+k_{1}\right)^{2}}-m q w & -\frac{c_{1} u}{\left(u+k_{1}\right)} & -m q u \\
\frac{c_{2} v^{2}}{\left(u+k_{2}\right)^{2}} & a_{2}-\frac{2 c_{2} v}{u+k_{2}} & 0 \\
\lambda p m q w & 0 & \lambda(p m q u-c)
\end{array}\right) .
$$

At $E_{0}=(0,0,0)$ the associated jacobian matrix is

$$
J\left(E_{0}\right)=\left(\begin{array}{ccc}
a_{1} & 0 & 0 \\
0 & a_{2} & 0 \\
0 & 0 & -\lambda c
\end{array}\right)
$$

Then, we find the following eigenvalues $a_{1}, a_{2}$ and $-\lambda c$. So, $E_{0}=(0,0,0)$ is unstable with $\operatorname{dim} W^{s}\left(E_{0}\right)=1$ and $\operatorname{dim} W^{u}\left(E_{0}\right)=2$.

At $E_{1}=\left(\frac{a_{1}}{b_{1}}, 0,0\right)$ the associated jacobian matrix is as follows:

$$
J\left(E_{1}\right)=\left(\begin{array}{ccc}
-a_{1} & -\frac{c_{1} a_{1}}{a_{1}+k_{1} b_{1}} & -m q \frac{a_{1}}{b_{1}} \\
0 & a_{2} & 0 \\
0 & 0 & \lambda\left(\frac{a_{1}}{b_{1}} p m q-c\right)
\end{array}\right) .
$$

The associated characteristic equation is given by

$$
\left(-a_{1}-x\right)\left(a_{2}-x\right)\left(\lambda\left(\frac{a_{1}}{b_{1}} p m q-c\right)-x\right)=0 .
$$

Then, we find the corresponding eigenvalues $-a_{1}, a_{2}$ and $\lambda\left(\frac{a_{1}}{b_{1}} p m q-c\right)$. So, $E_{1}=\left(\frac{a_{1}}{b_{1}}, 0,0\right)$ is unstable with $\operatorname{dim} W^{S}\left(E_{1}\right)=1$ and $\operatorname{dim} W^{u}\left(E_{1}\right)=2$.

At $E_{2}=\left(0, \frac{a_{2} k_{2}}{c_{2}}, 0\right)$ the jacobian matrix reads as:

$$
J\left(E_{2}\right)=\left(\begin{array}{ccc}
a_{1}-\frac{c_{1} a_{2} k_{2}}{k_{1} c_{2}} & 0 & 0 \\
\frac{a_{2}^{2}}{c_{2}} & -a_{2} & 0 \\
0 & 0 & -\lambda c
\end{array}\right) .
$$

The characteristic equation is as follows

$$
\left(a_{1}-\frac{c_{1} a_{2} k_{2}}{k_{1} c_{2}}-x\right)\left(a_{2}+x\right)(\lambda c+x)=0 .
$$

Then, we find the corresponding eigenvalues $a_{1}-\frac{c_{1} a_{2} k_{2}}{k_{1} c_{2}},-a_{2}$ and $-\lambda c$. Consequently, $E_{2}=\left(0, \frac{a_{2} k_{2}}{c_{2}}, 0\right)$ is locally asymptotically stable if $c_{1} a_{2} k_{2}>a_{1} k_{1} c_{2}$. 
At $E_{3}=\left(\widehat{u_{3}}, \widehat{v_{3}}, 0\right)$ the associated jacobian matrix is

$$
J\left(E_{3}\right)=\left(\begin{array}{ccc}
a_{1}-2 b_{1} u-\frac{c_{1} k_{1} \widehat{v_{3}}}{\left(\widehat{\left.u_{3}+k_{1}\right)^{2}}\right.} & -\frac{c_{1} \widehat{u_{3}}}{\left(\widehat{u_{3}}+k_{1}\right)} & -m q \widehat{u_{3}} \\
\frac{a_{2}^{2}}{c_{2}} & -a_{2} & 0 \\
0 & 0 & \lambda\left(p m q \widehat{u_{3}}-c\right)
\end{array}\right) .
$$

The characteristic equation is

$$
\left(\lambda\left(p m q \widehat{u_{3}}-c\right)-x\right)\left(x^{2}+\left(a_{2}-a_{1}+2 b_{1} u+\frac{c_{1} k_{1} \widehat{v_{3}}}{\left(\widehat{u_{3}}+k_{1}\right)^{2}}\right) x-a_{2}\left(a_{1}-2 b_{1} u-\frac{c_{1} k_{1} \widehat{v_{3}}}{\left(\widehat{u_{3}}+k_{1}\right)^{2}}\right)+\frac{c_{1} \widehat{u_{3}}}{\left(\widehat{u_{3}}+k_{1}\right)}\right)=0 .
$$

If $p m q \widehat{u_{3}}>c$, then $E_{3}=\left(\widehat{u_{3}}, \widehat{v_{3}}, 0\right)$ is unstable.

At $E_{4}=\left(\frac{c}{p m q}, 0, \frac{a_{1} p m q-b_{1} c}{p m^{2} q^{2}}\right)$ the jacobian matrix is

$$
J\left(E_{4}\right)=\left(\begin{array}{ccc}
-\frac{b_{1} c}{p m q} & -\frac{c_{1} c}{p m q k_{1}+c} & -\frac{c}{p} \\
0 & a_{2} & 0 \\
\frac{\lambda\left(a_{1} p m q-b_{1} c\right)}{m q} & 0 & 0
\end{array}\right) .
$$

The characteristic equation is

$$
\left(a_{2}-x\right)\left(x^{2}+\frac{b_{1} c}{p m q} x+\frac{\lambda c\left(a_{1} p m q-c b_{1}\right)}{p m q}\right)=0 .
$$

Then, we find the following eigenvalues $a_{2}>0$ and $x_{1}, x_{2}$, where $x_{1}=$ $\frac{1}{2}\left(\frac{b_{1} c}{p m q}+\sqrt{\left(\frac{b_{1} c}{p m q}\right)^{2}-4 \frac{\lambda c\left(a_{1} p m q-c b_{1}\right)}{p m q}}\right), \quad x_{2} \quad=\quad \frac{1}{2}\left(\frac{b_{1} c}{p m q}-\sqrt{\left(\frac{b_{1} c}{p m q}\right)^{2}-4 \frac{\lambda c\left(a_{1} p m q-c b_{1}\right)}{p m q}}\right) \quad$ if $\left(\frac{b_{1} c}{p m q}\right)^{2}-4 \frac{\lambda c\left(a_{1} p m q-c b_{1}\right)}{p m q}>0$ and $x_{1}=\frac{1}{2}\left(\frac{b_{1} c}{p m q}+i \sqrt{-\left(\frac{b_{1} c}{p m q}\right)^{2}+4 \frac{\lambda c\left(a_{1} p m q-c b_{1}\right)}{p m q}}\right), x_{2}=$ $\frac{1}{2}\left(\frac{b_{1} c}{p m q}-i \sqrt{-\left(\frac{b_{1} c}{p m q}\right)^{2}+4 \frac{\lambda c\left(a_{1} p m q-c b_{1}\right)}{p m q}}\right)$ if $\left(\frac{b_{1} c}{p m q}\right)^{2}-4 \frac{\lambda c\left(a_{1} p m q-c b_{1}\right)}{p m q}<0$. So $E_{4}=\left(\frac{c}{p m q}, 0, \frac{a_{1} p m q-b_{1} c}{p m^{2} q^{2}}\right)$ is unstable.

\section{Interior equilibrium and stability}

In this section, we discuss the local and global stability of the positive steady state $E^{\star}=\left(u^{\star}, v^{\star}, w^{\star}\right)$ of system (1.4).

Lemma 3.1. If the following hypotheses

$$
\begin{gathered}
\left(H_{1}\right): a_{1} p m q>b_{1} c, \\
\left(H_{2}\right): \frac{a_{1} p m q-b_{1} c}{p m q}>\frac{c_{1} a_{2}\left(c+k_{2} p m q\right)}{c_{2}\left(c+k_{1} p m q\right)}
\end{gathered}
$$

and

$$
\left(H_{3}\right): \frac{a_{1} p m q-b_{1} c}{p m q}-\frac{c_{1} a_{2}\left(c+k_{2} p m q\right)}{c_{2}\left(c+k_{1} p m q\right)}<m q
$$

are satisfied. Then system (1.4) has a positive equilibrium $E^{\star}=\left(u^{\star}, v^{\star}, w^{\star}\right)$, with

$$
u^{\star}=\frac{c}{p m q}, v^{\star}=\frac{a_{2}}{c_{2}}\left(u^{\star}+k_{2}\right), w^{\star}=\frac{1}{m q}\left(\frac{a_{1} p m q-b_{1} c}{p m q}-\frac{c_{1} a_{2}\left(c+k_{2} p m q\right)}{c_{2}\left(c+k_{1} p m q\right)}\right) .
$$


Remark 3.1. The hypothesis $\left(H_{1}\right)$ guarantees the existence of the interior equilibrium $E^{\star}$ exists, but does not guarantee the boundedness of positives solutions (see Theorem 2.1).

Theorem 3.1. If $E^{\star}=\left(u^{\star}, v^{\star}, w^{\star}\right)$ exists and

$$
\begin{gathered}
a_{1}<a_{2}+2 b u^{*}+M_{1}+m q w^{*}, \\
a_{2} a_{1}^{2}+M_{6} a_{1}+M_{7}>0,
\end{gathered}
$$

then $E^{\star}=\left(u^{\star}, v^{\star}, w^{\star}\right)$ is locally asymptotically stable.

Proof. We denote $E=(u, v, w)^{T}$ and

$$
L(E)=\left(\begin{array}{c}
f(u, v, w) \\
g(u, v, w) \\
h(u, v, w)
\end{array}\right)=\left(\begin{array}{c}
\left(a_{1}-b_{1} u-\frac{c_{1} v}{u+k_{1}}\right) u-m q u w \\
\left(a_{2}-\frac{c_{2} v}{u+k_{2}}\right) v \\
\lambda(p m q u-c) w
\end{array}\right),
$$

system (1.4) can be written as:

$$
\frac{d E}{d t}=L(E)
$$

Define $L_{E}\left(E^{\star}\right)$ as

$$
L_{E}\left(E^{\star}\right)=\left(\begin{array}{lll}
a_{11} & a_{12} & a_{13} \\
a_{21} & a_{22} & a_{23} \\
a_{31} & a_{32} & a_{33}
\end{array}\right)
$$

where

$$
\left\{\begin{array}{l}
a_{11}=a_{1}-2 b_{1} u^{\star}-\frac{c_{1} k_{1} v^{\star}}{\left(u^{*}+k_{1}\right)^{2}}-m q w^{\star}, a_{12}=-\frac{c_{1} u^{*}}{\left(u^{*}+k_{1}\right)}, a_{13}=-m q u^{\star}, \\
a_{21}=\frac{a_{2}^{2}}{c_{2}}, a_{22}=-a_{2}, a_{23}=0, \\
a_{31}=\lambda p m q w^{\star}, a_{32}=0, a_{33}=\lambda\left(p m q u^{\star}-c\right) .
\end{array}\right.
$$

The characteristic polynomial of $L_{E}\left(E^{*}\right)$ is given by

$$
\varphi(x)=x^{3}+B_{1} x^{2}+B_{2} x+B_{3} .
$$

With

$$
\begin{gathered}
B_{1}=a_{2}-a_{1}+2 b_{1} u^{\star}+\frac{c_{1} k_{1} v^{\star}}{\left(u^{\star}+k_{1}\right)^{2}}+m q w^{\star}, \\
B_{2}=-a_{2} a_{1}+2 a_{2} b_{1} u^{\star}+\frac{a_{2} c_{1} k_{1} v^{\star}}{\left(u^{\star}+k_{1}\right)^{2}}+a_{2} m q w^{\star}+w^{\star} \lambda u^{\star} p m^{2} q^{2}+\frac{a_{2}^{2} c_{1} u^{\star}}{c_{2}\left(u^{\star}+k_{1}\right)}, \\
B_{3}=w^{\star} \lambda u^{\star} p m^{2} q^{2} a_{2} .
\end{gathered}
$$

The condition (3.4) implies that $B_{1}>0$ and $B_{3} \geq 0$.

$$
B_{1} B_{2}-B_{3}=\left(a_{2}-a_{1}+M_{2}+M_{1}+M_{3}\right)\left(-a_{2} a_{1}+2 a_{2} M_{2}+a_{2} M_{1}+a_{2} M_{3}+M_{4}+a_{2}^{2} M_{5}\right)-a_{2} M_{4},
$$

where

$$
M_{1}=\frac{c_{1} k_{1} v^{\star}}{\left(u^{\star}+k_{1}\right)^{2}}, M_{2}=2 b_{1} u^{\star}, M_{3}=m q w^{\star}, M_{4}=w^{\star} \lambda u^{\star} p m^{2} q^{2}, M_{5}=\frac{c_{1} u^{\star}}{c_{2}\left(u^{\star}+k_{1}\right)} .
$$


by a simple computation, we obtain

$$
B_{1} B_{2}-B_{3}=a_{2} a_{1}^{2}+M_{6} a_{1}+M_{7}
$$

Where

$$
\begin{gathered}
M_{6}=-\left(a_{2}^{2}+M_{4}+a_{2}\left(2 M_{3}+2 M_{1}+3 M_{2}+a_{2} M_{5}\right)\right) . \\
M_{7}=a_{2}^{3} M_{5}+a_{2}^{2}\left(2 M_{2}+M_{1}+M_{3}+M_{3} M_{5}+M_{1} M_{5}+M_{5} M_{2}\right)+a_{2}\left(2 M_{2}^{2}+3 M_{1} M_{2}+3 M_{2} M_{3}+M_{1}^{2}+M_{3}^{2}+2 M_{1} M_{3}\right) \\
+M_{1} M_{4}+M_{2} M_{4}+M_{3} M_{4} .
\end{gathered}
$$

From the expression (3.5), we find $B_{1} B_{2}-B_{3}>0$. By applying the Routh Hurwitz criterion, $E^{\star}=\left(u^{\star}, v^{\star}, w^{\star}\right)$ is locally asymptotically stable.

Theorem 3.2. If the conditions of the lemma (3.1) are satisfied and if

$$
\frac{b_{1}}{c_{1}}>\frac{v^{\star}}{\left(u^{\star}+k_{1}\right)\left(u+k_{1}\right)} \text {. }
$$

Then, the positive equilibrium $E^{\star}=\left(u^{\star}, v^{\star}, w^{\star}\right)$ is globally asymptotically stable.

Proof. Let us consider the following Lyapunov function,

$$
V(u, v, w)=h_{1}(u)+h_{2}(v)+h_{3}(w)
$$

where $h_{1}(u)=T_{1} \int_{u^{*}}^{u} \frac{\left(\eta-u^{*}\right)}{\eta} d \eta, h_{2}(v)=T_{2} \int_{v^{*}}^{v} \frac{\left(\eta-v^{*}\right)}{\eta} d \eta$ and $h_{3}(w)=T_{3} \int_{w^{*}}^{w} \frac{\left(\eta-w^{*}\right)}{\eta} d \eta$.

Then, we have $V\left(u^{\star}, v^{\star}, w^{\star}\right)=0$ and $V(u, v, w)>0$ for all $(u, v, w) \in \mathbb{R}^{3+} \backslash\left\{\left(u^{\star}, v^{\star}, w^{\star}\right)\right\}$. The time derivative of $V$ along the solution of system (1.4), we have

$$
\frac{d V}{d t}=T_{1}\left(u-u^{\star}\right)\left[a_{1}-b_{1} u-\frac{c_{1} v}{u+k_{1}}-m q w\right]+T_{2}\left(v-v^{\star}\right)\left[a_{2}-\frac{c_{2} v}{u+k_{2}}\right]+T_{3}\left(w-w^{\star}\right)[\lambda(p m q u-c)] .
$$

$T_{1}, T_{2}$, and $T_{3}$ are positive constants to be determined in the subsequent steps, using the following expressions

$$
a_{1}=b_{1} u^{\star}+\frac{c_{1} v^{\star}}{u^{\star}+k_{1}}+m q w^{\star}, a_{2}=\frac{c_{2} v^{\star}}{u^{\star}+k_{2}}, c=p m q u^{\star},
$$

we have

$$
\begin{aligned}
\frac{d V}{d t} & =T_{1}\left(u-u^{\star}\right)\left[-b_{1}\left(u-u^{\star}\right)-\frac{c_{1}\left(v-v^{\star}\right)}{\left(u+k_{1}\right)}+\frac{c_{1} v^{\star}\left(u-u^{\star}\right)}{\left(u+k_{1}\right)\left(u^{\star}+k_{1}\right)}-m q\left(w-w^{\star}\right)\right] \\
& +T_{2}\left(v-v^{\star}\right)\left[\frac{c_{2} v^{\star}\left(u-u^{\star}\right)}{\left(u+k_{2}\right)\left(u^{\star}+k_{2}\right)}-\frac{c_{2}\left(v-v^{\star}\right)}{\left(u+k_{2}\right)}\right]+T_{3} \lambda p m q\left(w-w^{\star}\right)\left(u-u^{\star}\right) \\
& =T_{1}\left(u-u^{\star}\right)^{2}\left[-b_{1}+\frac{c_{1} v^{\star}}{\left(u+k_{1}\right)\left(u^{\star}+k_{1}\right)}\right]-\frac{T_{2} c_{2}}{\left(u+k_{2}\right)}\left(v-v^{\star}\right)^{2}+\left(u-u^{\star}\right)\left(v-v^{\star}\right)\left[\frac{-T_{1} c_{1}}{\left(u+k_{1}\right)}+\frac{T_{2} c_{2} v^{\star}}{\left(u+k_{2}\right)\left(u^{\star}+k_{2}\right)}\right] \\
& +\left(u-u^{\star}\right)\left(w-w^{\star}\right)\left(T_{3} \lambda p m q-T_{1} m q\right) \\
& \leq T_{1}\left(u-u^{\star}\right)^{2}\left[-b_{1}+\frac{c_{1} v^{\star} b_{1}}{\left(a_{1}+b_{1} k_{1}\right)\left(u^{\star}+k_{1}\right)}\right]-\frac{T_{2} c_{2} b_{1}}{\left(a_{1}+b_{1} k_{2}\right)}\left(v-v^{\star}\right)^{2} \\
& +\left(u-u^{\star}\right)\left(v-v^{\star}\right)\left[\frac{-T_{1} c_{1} b_{1}}{\left(a_{1}+b_{1} k_{1}\right)}+\frac{T_{2} a_{2} b_{1}}{\left(a_{1}+b_{1} k_{2}\right)}\right]+\left(u-u^{\star}\right)\left(w-w^{\star}\right)\left(T_{3} \lambda p m q-T_{1} m q\right) .
\end{aligned}
$$

and choosing

$$
T_{1}=1, T_{2}=\frac{c_{1}\left(a_{1}+b_{1} k_{2}\right)}{\left(a_{1}+b_{1} k_{1}\right) a_{2}}, T_{3}=\frac{1}{\lambda p} .
$$


Then, we deduce that:

$$
\frac{d V}{d t} \leq\left(u-u^{\star}\right)^{2}\left[-b_{1}+\frac{c_{1} v^{\star} b_{1}}{\left(a_{1}+b_{1} k_{1}\right)\left(u^{\star}+k_{1}\right)}\right]-\frac{c_{1} c_{2} b_{1}}{a_{2}\left(a_{1}+b_{1} k_{1}\right)}\left(v-v^{\star}\right)^{2} .
$$

From the expression (3.11), the coefficients of $\left(u-u^{*}\right)^{2}$ and $\left(v-v^{*}\right)^{2}$ are negative. Then we deduce that $\frac{d V}{d t}<0$ and $\frac{d V}{d t}=0$ if and only if $u=u^{\star}, v=v^{\star}$ and $w=w^{\star}$. Then, the function $V$ satisfy the Lyapunov conditions and by LaSalle's Theorem [10], $E^{\star}=\left(u^{\star}, v^{\star}, w^{\star}\right)$ is globally asymptotically stable.

In the next, we will establish the existence of periodic oscillations around the interior equilibrium via Hopf bifurcation Theorem.

\section{Bifurcation analysis}

Choosing $a_{1}$ as a parameter of bifurcation, then we have the following result on the existence of bifurcating branch of periodic solutions.

Theorem 4.1. Suppose that $\left(H_{i}\right) i=1,2,3$ are satisfied and $a_{1}<a_{2}+2 b u^{\star}+M_{1}+m q w^{\star}$ and $M_{6}^{2} \geq 4 a_{2} M_{7}$, then a Hopf bifurcation occurs at the unique positive value

$$
a_{1}=a_{1 c}=\frac{-M_{6}-\sqrt{M_{6}^{2}-4 a_{2} M_{7}}}{2 a_{2}} .
$$

Proof. The characteristic equation associated to $E^{\star}=\left(u^{\star}, v^{\star}, w^{\star}\right)$ is given by

$$
\varphi(x)=x^{3}+B_{1}\left(a_{1}\right) x^{2}+B_{2}\left(a_{1}\right) x+B_{3}\left(a_{1}\right) .
$$

The necessary and sufficient conditions of Hopf bifurcation to occur are: there exists $a_{1}=a_{1 c}$ such that

$$
\text { (a) } B_{i}\left(a_{1 c}\right)>0, i=1,2,3 \text {, }
$$

(b) $B_{1}\left(a_{1 c}\right) B_{2}\left(a_{1 c}\right)-B_{3}\left(a_{1 c}\right)=0$,

and

$$
\text { (c) } \operatorname{Re}\left[\frac{d x_{j}}{d a_{1}}\right]_{a_{1}=a_{1 c}} \neq 0, i=1,2,3 \text {. }
$$

The condition $B_{1} B_{2}-B_{3}$ gives

$$
B_{1} B_{2}-B_{3}=a_{2} a_{1}^{2}+M_{6} a_{1}+M_{7}=0 .
$$

Since $B_{2}>0$ at $a_{1}=a_{1 c}$, there exists an interval $\left(a_{1 c}-\epsilon, a_{1 c}+\epsilon\right)$ containing $a_{1}$, for each $\epsilon>0$. Therefore, for $a_{1} \in\left(a_{1 c}-\epsilon, a_{1 c}+\epsilon\right)$ the characteristic equation cannot have roots with containing negative real parts. For $a_{1}=a_{1 c}$, we have

$$
\left(x^{2}+B_{2}\right)\left(x+B_{1}\right)=0,
$$

which has three roots $x_{1}=i \sqrt{B_{2}}, x_{2}=-i \sqrt{B_{2}}$ and $x_{3}=-B_{1}$. For $a_{1} \in\left(a_{1 c}-\epsilon, a_{1 c}+\epsilon\right)$, the roots take the following general form:

$$
\begin{gathered}
x_{1}\left(a_{1}\right)=\alpha_{1}\left(a_{1}\right)+i \alpha_{2}\left(a_{1}\right), \\
x_{2}\left(a_{1}\right)=\alpha_{1}\left(a_{1}\right)-i \alpha_{2}\left(a_{1}\right), \\
x_{3}\left(a_{1}\right)=-B_{1}\left(a_{1}\right) .
\end{gathered}
$$


Now, we verify the transversality condition $\operatorname{Re}\left[\frac{d x_{i}}{d a_{1}}\right]_{a_{1}=a_{1 c}} \neq 0, i=1,2$, 3. Substituting $x_{1}\left(a_{1}\right)=\alpha_{1}\left(a_{1}\right)+$ $i \alpha_{2}\left(a_{1}\right)$ in (4.2) and calculating the derivative, we get

$$
\begin{aligned}
& \Omega_{1}\left(a_{1}\right) \alpha_{1}^{\prime}\left(a_{1}\right)-\Omega_{2}\left(a_{1}\right) \alpha_{2}^{\prime}\left(a_{1}\right)+N_{1}\left(a_{1}\right)=0, \\
& \Omega_{2}\left(a_{1}\right) \alpha_{1}^{\prime}\left(a_{1}\right)+\Omega_{1}\left(a_{1}\right) \alpha_{2}^{\prime}\left(a_{1}\right)+N_{2}\left(a_{1}\right)=0,
\end{aligned}
$$

where

$$
\begin{gathered}
\Omega_{1}\left(a_{1}\right)=3 \alpha_{1}^{2}\left(a_{1}\right)+2 B_{1}\left(a_{1}\right) \alpha_{1}\left(a_{1}\right)+\beta_{2}\left(a_{1}\right)-3 \alpha_{2}^{2}\left(a_{1}\right), \\
\Omega_{2}\left(a_{1}\right)=6 \alpha_{1}\left(a_{1}\right) \alpha_{2}\left(a_{1}\right)+2 B_{1}\left(a_{1}\right) \alpha_{2}\left(a_{1}\right), \\
N_{1}\left(a_{1}\right)=\alpha_{1}^{2}\left(a_{1}\right) B_{1}^{\prime}\left(a_{1}\right)+\beta_{2}^{\prime}\left(a_{1}\right) \alpha_{1}\left(a_{1}\right)+B_{3}^{\prime}\left(a_{1}\right)-\alpha_{2}^{2}\left(a_{1}\right) B_{1}^{\prime}\left(a_{1}\right), \\
N_{2}\left(a_{1}\right)=2 \alpha_{1}\left(a_{1}\right) \alpha_{2}\left(a_{1}\right) B_{1}^{\prime}\left(a_{1}\right)+B_{2}^{\prime}\left(a_{1}\right) \alpha_{2}\left(a_{1}\right) .
\end{gathered}
$$

Since $\Omega_{2}\left(a_{1 c}\right) N_{2}\left(a_{1 c}\right)+\Omega_{1}\left(a_{1 c}\right) N_{1}\left(a_{1 c}\right) \neq 0$, we have $\operatorname{Re}\left[\frac{d x_{i}}{d a_{1}}\right]_{a_{1}=a_{1 c}}=\left.\frac{\Omega_{1} N_{1}+\Omega_{2} N_{2}}{\Omega_{2}^{2}+\Omega_{1}^{2}}\right|_{a_{1}=a_{1 c}} \neq 0, i=1,2$ and $x_{3}\left(a_{1 c}\right)=-B_{1}\left(a_{1 c}\right)$.

Hence the proof of the theorem.

\section{Optimal Harvesting Policy}

In commercial exploitation of renewable resources, the fundamental problem from the economic point of view is to determine the optimal trade off between present and future harvests. In the prey-predator model, the biological resources are most likely to be harvested and sold with the purpose of achieving the economic interest which motivates the introduction of harvesting in the prey-predator model [19].

Our objective in this section, is to determine an optimal harvesting policy and to maximize the total return produced by the exploitation of the resources.

The optimal control problem is given by the value $J$, which presents a continuous time-stream of revenues [20].

$$
J=\int_{0}^{\infty} e^{-\delta t}(p m q u-c) w d t,
$$

where $\delta$ denotes the instantaneous annual rate of discount [21].

Thus, our goal is to maximize $J$ subject to the state equations (1.4) by using Pontryagin's maximum principle [22]. The convexity of the function $J$ with respect to $m$, the linearity of the differential equations in the control and the compactness of the range values of the state variables can be combined to give the existence of the optimal control.

We choose $m_{\delta}$ is an optimal control with corresponding states $u_{\delta}, v_{\delta}$ and $w_{\delta}$. We take $E_{\delta}=\left(u_{\delta}, v_{\delta}, w_{\delta}\right)$ as optimal equilibrium point. Here we intend to derive optimal control such that

$$
J\left(m_{\delta}\right)=\max \{J(m), m \in \Gamma\}
$$

where $\Gamma$ is the control set defined by

$$
\Gamma=\left\{m:\left[t_{0}, t_{f}\right] \rightarrow\left[m_{\min }, m_{\max }\right], \text { is lebesgue measurable }\right\} .
$$

The associated Hamiltonian function is given by:

$$
H=(p m q u-c) w+\lambda_{1}\left[\left(a_{1}-b_{1} u-\frac{c_{1} v}{u+k_{1}}\right) u-m q u w\right]+\lambda_{2}\left[\left(a_{2}-\frac{c_{2} v}{u+k_{2}}\right) v\right]+\lambda_{3}[\lambda(p m q u-c) w] .
$$

Where $\lambda_{1}, \lambda_{2}$, and $\lambda_{3}$ are the adjoint variables.

The transversality conditions give $\lambda_{i}\left(t_{f}\right)=0, i=1,2,3$. 
The control variable $m_{\delta}$ is subjected to the constraints $m_{\min } \leq m_{\delta}(t) \leq m_{\max }$ is the control set where $m_{\max }$ (resp $m_{\min }$ ) is a feasible upper limit for the harvesting effort (resp. feasible lower limit for the harvesting effort).

Here we treat with an optimal equilibrium solution. Since we are considering an equilibrium solution, $u_{\delta}, v_{\delta}$ and $w_{\delta}$ are to be used as constants in the subsequent steps.

By Pontryagin's maximum principle, the adjoint equations at the point $E_{\delta}=\left(u_{\delta}, v_{\delta}, w_{\delta}\right)$ are

$$
\begin{aligned}
\frac{d \lambda_{1}}{d t}=\delta \lambda_{1}-\left.\frac{\partial H}{\partial u}\right|_{E_{\delta}} & \\
=\delta \lambda_{1}-\left[p m q w_{\delta}+\lambda_{1}\right. & \left.\left(a_{1}-2 b_{1} u_{\delta}-\frac{c_{1} k_{1} v_{\delta}}{\left(u_{\delta}+k_{1}\right)^{2}}-m q w_{\delta}\right)+\lambda_{2}\left(\frac{c_{2} v_{\delta}^{2}}{\left(u_{\delta}+k_{2}\right)^{2}}\right)+\lambda_{3} p m q w_{\delta} \lambda\right] \\
\frac{d \lambda_{2}}{d t} & =\delta \lambda_{2}-\left.\frac{\partial H}{\partial v}\right|_{E_{\delta}} \\
& =\delta \lambda_{2}-\left[\lambda_{1}\left(-\frac{c_{1} u_{\delta}}{\left(u_{\delta}+k_{1}\right)}\right)+\lambda_{2}\left(a_{2}-\frac{2 c_{2} v_{\delta}}{u_{\delta}+k_{2}}\right)\right] \\
\frac{d \lambda_{3}}{d t} & =\delta \lambda_{3}-\left.\frac{\partial H}{\partial w}\right|_{E_{\delta}} \\
& =\delta \lambda_{3}-\left[\left(p m q u_{\delta}-c\right)-\lambda_{1} m q u_{\delta}+\lambda_{3}\left(p m q u_{\delta}-c\right) \lambda\right] .
\end{aligned}
$$

By direct calculation we have

$$
\begin{gathered}
\lambda_{1}=\frac{\frac{p m_{\delta} q w \delta}{\left(\delta-\left(p m_{\delta} q u_{\delta}-c\right) \lambda\right)}}{\left[\delta-a_{1}+2 b_{1} u_{\delta}+\frac{c_{1} k_{1} v_{\delta}}{\left(u_{\delta}+k_{1}\right)^{2}}+m_{\delta} q w_{\delta}\right]+\frac{c_{1} c_{2} u_{\delta} v_{\delta}^{2}}{\left(u_{\delta}+k_{1}\right)\left(u_{\delta}+k_{2}\right)\left[a_{2}\left(u_{\delta}+k_{2}\right)-c_{2} v_{\delta}-\delta\left(u_{\delta}+k_{2}\right)\right]}+\frac{p m_{\delta}^{2} q^{2} u_{\delta} w_{\delta} \lambda}{\left(\delta-\left(p m_{\delta} q u_{\delta}-c\right) \lambda\right)}}=A(\text { say }) \\
\lambda_{2}=-\frac{\frac{c_{1} u_{\delta}}{\left(u_{\delta}+k_{1}\right)}}{\left(a_{2}-\frac{c_{2} v_{\delta}}{u_{\delta}+k_{2}}\right)-\delta} A \\
\lambda_{3}=\frac{\left(p m_{\delta} q u_{\delta}-c\right)-A m_{\delta} q u_{\delta}}{\delta-\left(p m_{\delta} q u_{\delta}-c\right) \lambda}
\end{gathered}
$$

The Hamiltonian $H$ must be maximised for $m_{\delta} \in\left[m_{\min }, m_{\max }\right]$. With the control constraints $m_{\min } \leq m_{\delta}(t) \leq$ $m_{\max }$ are not binding (that is, the optimal equilibrium does not occur either at $m_{\delta}=m_{\min }$ or $m_{\delta}=m_{\max }$ ), we have singular control given by [18].

Then

$$
\frac{\partial H}{\partial m}=p q u_{\delta} w_{\delta}-\lambda_{1} q u_{\delta} w_{\delta}+\lambda_{3} \lambda p q u_{\delta} w_{\delta}=0 \text { at } m_{\delta}(t) .
$$

Now substituting the value of $\lambda_{1}$ and $\lambda_{3}$ into equation (5.12), we get

$$
p q u_{\delta} w_{\delta}-A q u_{\delta} w_{\delta}+\frac{\left(p m_{\delta} q u_{\delta}-c\right)-A m_{\delta} q u_{\delta}}{\delta-\left(p m_{\delta} q u_{\delta}-c\right) \lambda} \lambda p q u_{\delta} w_{\delta}=0 .
$$

Therefore

$$
m_{\delta}=\frac{p \delta^{2}+p c \delta \lambda N_{1}+\frac{1}{N_{2}} p c_{1} c_{2} \delta u_{\delta} v_{\delta}^{2}(\delta+c \lambda)}{p^{2} \delta \lambda q u_{\delta} N_{1}+\frac{1}{N_{2}} p^{2} q c_{1} c_{2} \lambda \delta u_{\delta}^{2} v_{\delta}^{2}}
$$

where

$$
N_{1}=\delta-a_{1}+2 b_{1} u_{\delta}+\frac{c_{1} k_{1} v_{\delta}}{\left(u_{\delta}+k_{1}\right)^{2}}
$$

and

$$
N_{2}=\left(u_{\delta}+k_{1}\right)\left(u_{\delta}+k_{2}\right)\left[a_{2}\left(u_{\delta}+k_{2}\right)-c_{2} v_{\delta}-\delta\left(u_{\delta}+k_{2}\right)\right] .
$$

Therefore, we summarize the above analysis by the following theorem: 
Theorem 5.1. There exist an optimal control $m_{\delta}$ and corresponding solutions $u_{\delta}, v_{\delta}$ and $w_{\delta}$ which maximize $J(m)$ over $\Gamma$. Furthermore, there exists adjoint functions $\lambda_{1}, \lambda_{2}$, and $\lambda_{3}$ satisfying equations (5.9) and (5.11) with transversality conditions $\lambda_{i}\left(t_{f}\right)=0, i=1,2,3$. Moreover, the optimal control is given by:

$$
m_{\delta}=\frac{p \delta^{2}+p c \delta \lambda N_{1}+\frac{1}{N_{2}} p c_{1} c_{2} \delta u_{\delta} v_{\delta}^{2}(\delta+c \lambda)}{p^{2} \delta \lambda q u_{\delta} N_{1}+\frac{1}{N_{2}} p^{2} q c_{1} c_{2} \lambda \delta u_{\delta}^{2} v_{\delta}^{2}} .
$$

\section{Numerical examples}

In this section, we present some numerical simulations with hypothetical set of parameters to understanding the theoretical results which have been showed in the previous sections of this work.

With the following parameters:

$$
b_{1}=0.01, k_{1}=0.6, k_{2}=0.1, a_{2}=0.7, c_{1}=0.6, c_{2}=0.8, m=0.6, q=0.9, \lambda=1.5, p=0.9, c=0.7
$$

Firstly from the Figure (1) and (2), we observe that populations of prey-predator and harvesting effort con-

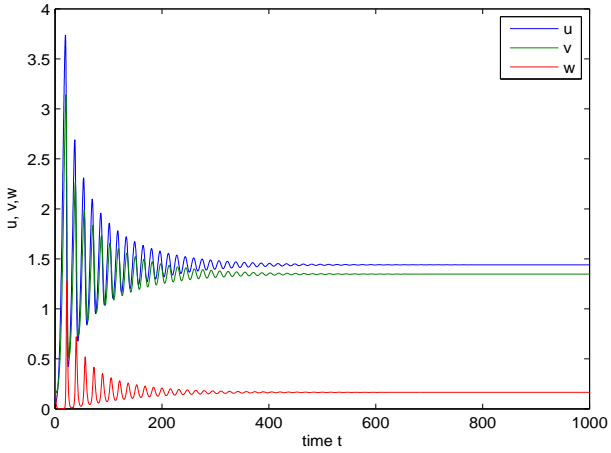

Figure 1: Stable behavior of prey-predator populations and harvesting effort with time for $a_{1}=0.5$.

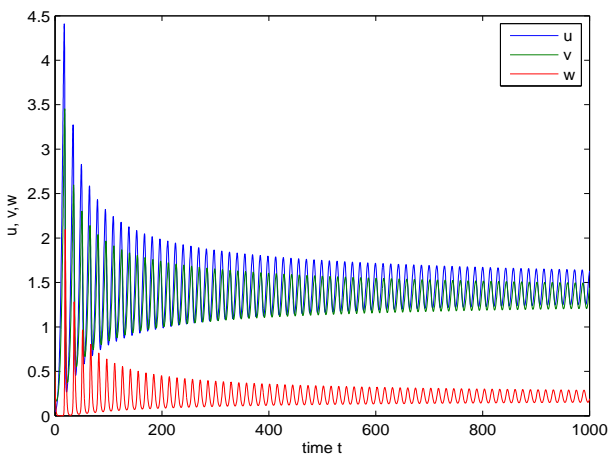

Figure 3: Solution curves of prey-predator populations and harvesting effort with the time for bifurcation parameter $a_{1}=$ 0.52489 .

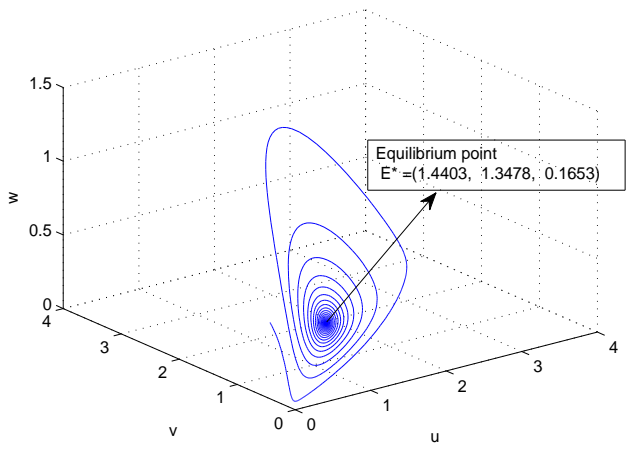

Figure 2: Phase space trajectories of $u, v, w$ corresponding to $a_{1}=0.5$.

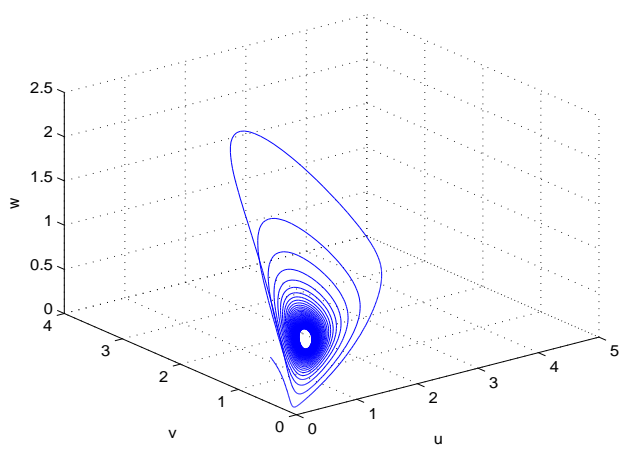

Figure 4: Phase space trajectories of $u, v, w$ corresponding to the bifurcation parameter $a_{1}=0.52489$. 


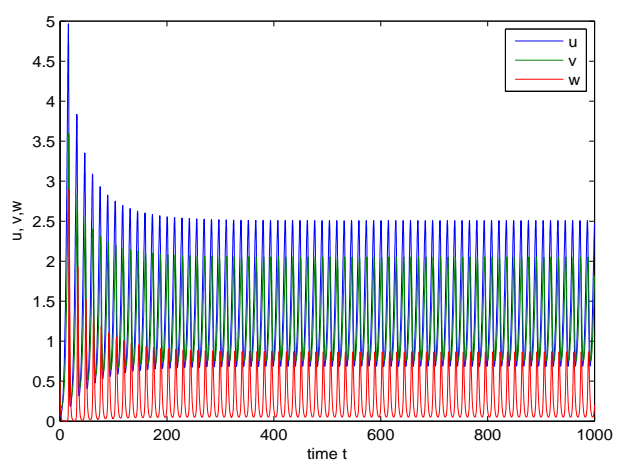

Figure 5: Solution curves of prey-predator populations and harvesting effort with the time for bifurcation parameter $a_{1}=0.55$.

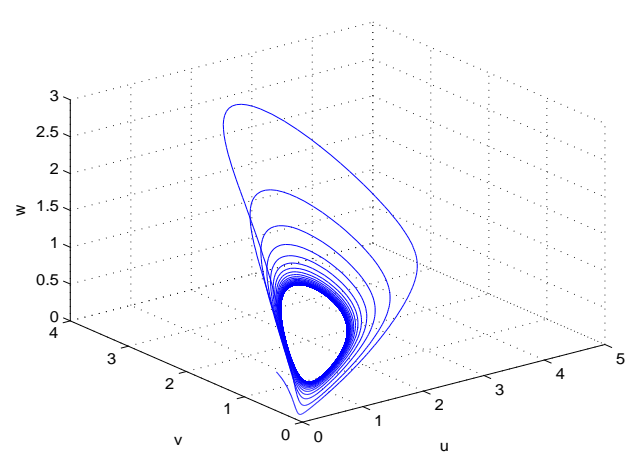

Figure 6: Phase space trajectories of $u, v, w$ corresponding to the bifurcation parameter $a_{1}=0.55$.

verge to their steady states with the passage of time and $E^{\star}=\left(u^{\star}, v^{\star}, w^{\star}\right)$ is locally asymptotically stable for system (1.4). If we increase the value of the bifurcation parameter $a_{1}=0.52489$ then Figures (3) and (4) show that equilibrium $E^{\star}=\left(u^{\star}, v^{\star}, w^{\star}\right)$ loses its stability and becomes unstable and there is a periodic orbit if $a_{1}=0.55$ Figures (5) and (6).

In the next, we study the variation of the prey, predator population and harvesting effort with time for different price per unit biomass of landed fish $p$ and the parameters are fixed as follows:

$a_{1}=0.5, b_{1}=0.01, k_{1}=0.6, k_{2}=0.1, a_{2}=0.7, c_{1}=0.6, c_{2}=0.8, m=0.7, q=0.09, \lambda=1.5, c=0.1$.

From Figure (7), we observe that prey and predator populations decreases with the increase of price value $p$.

Table 1: The table shows the values of parameters model.

\begin{tabular}{l|cccccccccccc}
\hline value of & $b_{1}$ & $k_{1}$ & $k_{2}$ & $a_{2}$ & $c_{1}$ & $c_{2}$ & $a_{1}$ & $q$ & $\lambda$ & $p$ & $c$ & $\delta$ \\
\hline Example 1 & 0.01 & 0.9 & 0.05 & 0.7 & 0.06 & 0.8 & 0.5 & 0.09 & 0.5 & 0.5 & 0.7 & 0.1 \\
Example 2 & 0.01 & 0.9 & 0.05 & 0.7 & 0.06 & 0.8 & 0.5 & 0.09 & 0.5 & 0.5 & 0.7 & 0.03 \\
\hline
\end{tabular}

Theorem (5.1) giving existence of the optimal equilibrium and by using the parameters values in table 1 and from optimal harvesting policy, we have proved the optimal value of $m_{\delta}$ and the corresponding optimal equilibrium $E_{\delta}$. Thus we deduce this table 2:

Table 2

\begin{tabular}{l|ccc}
\hline value of & $m_{\delta}$ & $E_{\delta}=\left(u_{\delta}, v_{\delta}, w_{\delta}\right)$ & Contour pictures \\
\hline Example 1 & 0.6110 & $(25.4592,22.3205,3.5388)$ & Figs. 8, 9 \\
Example 2 & 0.9262 & $(16.7950,14.7394,3.3839)$ & Figs. 10, 11 \\
\hline
\end{tabular}



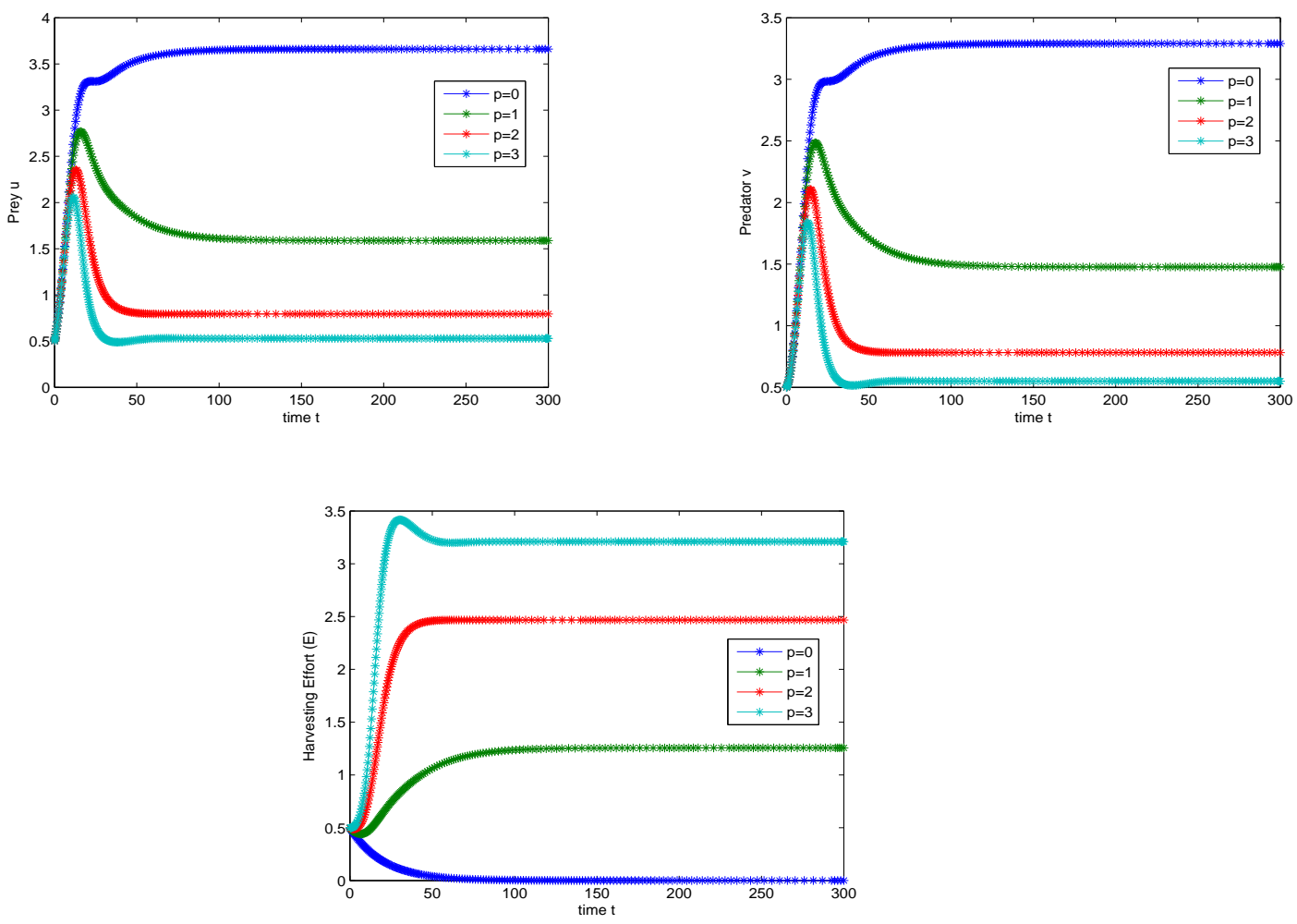

Figure 7: Variation of the prey-predator populations and harvesting effort with time for different price levels $p$.

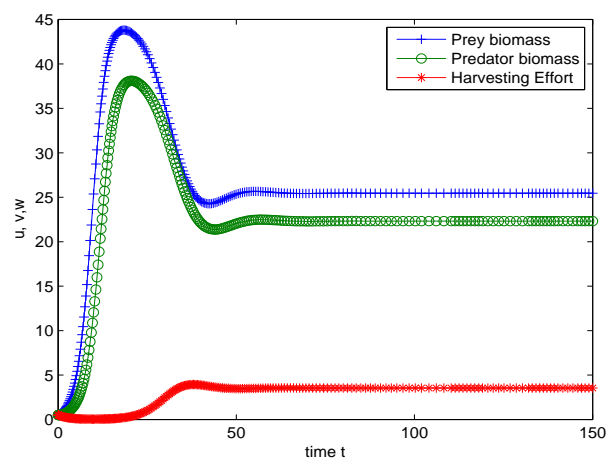

Figure 8: Variation of prey and predator biomass and harvesting effort with the increasing time when $m_{\delta}=0.6110$ and other parameters are shown in the Table 1. 
DE GRUYTER

Dynamics Analysis and Optimality in Selective Harvesting Predator-Prey Model $\longrightarrow \mathbf{1 5}$

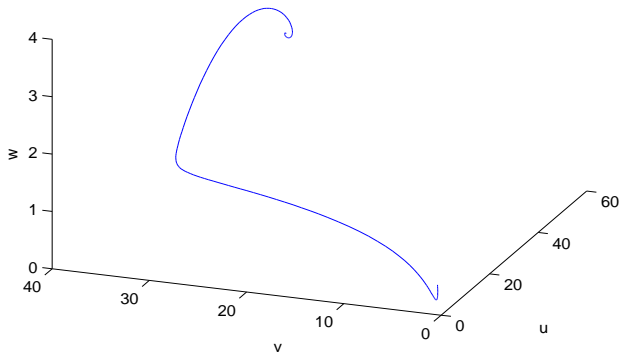

Figure 9: Phase portrait ( $u-v-w$ space) of model system (1.4) when $m_{\delta}=0.6110$ and other parameters are shown in the Table 1.

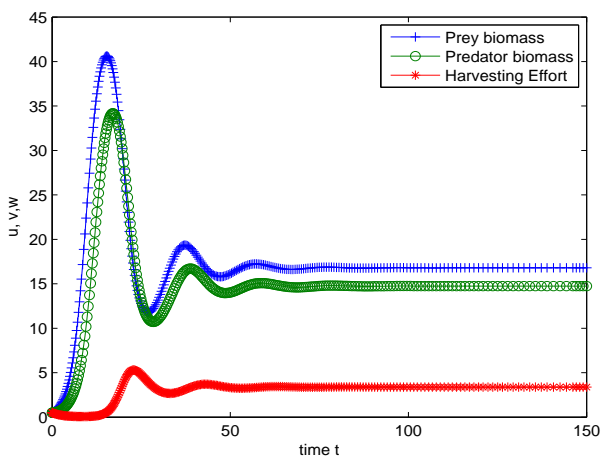

Figure 10: Variation of prey and predator biomass and harvesting effort with the increasing time when $m_{\delta}=0.9262$.

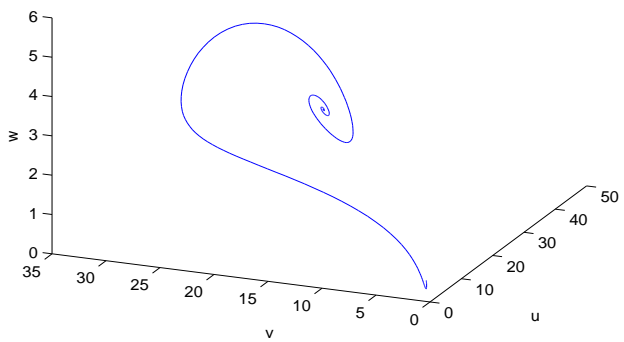

Figure 11: Phase portrait ( $u-v-w$ space) of model system (1.4) when $m_{\delta}=0.9262$. 


\section{References}

[1] C.W. Clark, Mathematical Bioeconomics: The Optimal Management of Renewal Resources, Wiley, New York, 1976.

[2] M. Fan, K. Wang, Optimal harvesting policy for single population with periodic coefficients, Math. Biosci. 152 (1998) 165-177.

[3] H. Qiu, J. Lv, K. Wang, The optimal harvesting policy for non-autonomous populations with discount, Appl. Math. Lett. 26 (2013) 244-248.

[4] E. Braverman, R. Mamdani, Continuous versus pulse harvesting for population models in constant and variable environment, J. Math. Biol. 57 (2008)413-434.

[5] M.A. Aziz-Alaoui and M. Daher Okiye, Boundedness and global stability for a predator-prey model with modified LeslieGower and Holling type II shemes, Applied Math. Let., 16, (2003), 1069-1075.

[6] M. Daher Okiye, Study and asymptotic analysis of some nonLinear dynamical systems : Application to predator-prey problems, in french, PHD Thesis, Le Havre University, France, 2004.

[7] M. A. Aziz-Alaoui, Study of a Leslie-Gower-type tritrophic population model, Chaos, Solitons and Fractals, 14 (8), (2002), 1275-1293.

[8] T.K. Kar, Modelling and analysis of a harvested prey-predator system incorporating a prey refuge, J. Comput. Appl. Math. 185 (2006) 19-33.

[9] D. Pal, G.S. Mahaptra, G.P. Samanta, Optimal harvesting of prey-predator system with interval biological parameters: a bioeconomic model, Math.Biosci. 241 (2013) 181-187.

[10] J. Hale, Ordinary Differential Equations, Krieger Publ. Co., Malabar, 1980.

[11] Kalyan Das, M.N. Srinivas, M.A.S. Srinivas c, N.H. Gazi, d Chaotic dynamics of a three species prey-predator competition model with bionomic harvesting due to delayed environmental noise as external driving force C. R. Biologies 335 (2012) 503-513.

[12] Manju Agarwal and Rachana Pathak, Persistence and optimal harvesting of prey-predator model with Holling Type III functional response, International Journal of Engineering, Science and Technology Vol. 4, No. 3, (2012) pp. 78-96.

[13] Xiao D, Ruan S. Bogdanov-Takens bifurcations in predator-prey systems with constant rate harvesting. Fields Inst Commun (1999)21:493-506.

[14] Myerscough MR, Gray BF, Hogarth WL, Norbury J. An analysis of an ordinary differential equations model for a two species predator-prey system with harvesting and stocking. J Math Biol (1992) 30:389-411.

[15] Brauer F, Soudack AC. Stability regions and transition phenomena for harvested predator-prey systems. J Math Biol (1979) 7:319-37.

[16] M.I.S. Costa, E. Kaszkurewicz, A. Bhaya, L. Hsu, Achieving global convergence to an equilibrium population in predator-prey systems by the use of a discontinuous harvesting policy, Ecol. Model. 128 (2000) 89.

[17] Chen, FD: On a nonlinear non-autonomous predator-prey model with diffusion and distributed delay. J. Comput. Appl. Math. 180, 33-49 (2005).

[18] J. Rebaza, Dynamics of prey threshold harvesting and refuge, J. Comput. Appl. Math. 236 (2012) 1743.

[19] S. Sarwardi, M. Haque, P.K. Mandal, Ratio-dependent predator-prey model of interactin population with delay effect, Nonlinear Dyn. 69 (2012) 817-836.

[20] K. Chakraborty, S. Jana, T.K. Kar, Global dynamics and bifurcation in a stage structured prey-predator fishery model with harvesting, Appl. Math.Comput. 218 (2012) 9271-9290.

[21] D. L. Ragozin and G. Brown, "Harvest policies and nonmarket valuation in a predatorprey system", J. Envirn. Econ. Manag. 12 (1985) 155-168.

[22] L. S. Pontryagin, V. G. Boltyonskü, R. V. Gamkrelidre and E. F. Mishchenko, The mathematical theory of optimal processes, Wiley, New York, 1962.

[23] T. Das, R.N. Mukherjee, K.S. Chaudhuri, Harvesting of a prey-predator fishery in the presence of toxicity, Appl. Math. Model. 33 (2009) 2282.

[24] M. Liu, K. Wang, Analysis of a stochastic autonomous mutualism model, J. Math. Anal. Appl. 402 (2013) 392-403.

[25] J.R. Beddington, R.M. May, Harvesting natural populations in a randomly fluctuating environment, Science 197 (1977) 463-465.

[26] W. Li, K. Wang, Optimal harvesting policy for general stochastic logistic population model, J. Math. Anal. Appl. 368 (2010) 420-428.

[27] A.R. Palma, E.G. Olivares, Optimal harvesting in a predator-prey model with Allee effect and sigmoid functional response, Appl. Math. Model. 5 (2012) 1864.

[28] C. Chen, C. Hsui, Fishery policy when considering the future opportunity of harvesting, Math. Biosci. 207 (2007) 138.

[29] Dubey, B.P. Chandra, et al. A model for fishery resourse with reserve area. Nonlinear Analysis : Real World Applications., 4(4) (2003). 625-637.

[30] K. R Fister and S. Lenhart, optimal harvesting in an age-structured predator-prey model, Appl. Math. Optim. 54 (2006) 1-15.

[31] Zhang X, chen L, Neumann UA (2000) The stage structured predator prey model and optimal harvesting policy. Math Biosci $168: 201-210$.

[32] Hsu, S.B. and Hwang T.W., Global stability for a class of predator- prey systems, SIAM J. Appl. Math., 55(3), (1995) $763-789$. 
[33] Hsu, S.B. and Hwang T.W., Uniqueness of limit cycles for a predator-prey system of Holling and Leslie type. Can. Appl. Math. Q., 6(2), 91-117, (1998).

[34] Daher, O. M. and Aziz-Alaoui, M. On the dynamics of a predator-prey model with the Holling-Tanner functional Editor V. Capasso, Proc. ESMTB conf, (2002) 270-278.

[35] R. Yafia, F. El Adnani and H. Talibi Limit cycle and numerical similations for small and large delays in a predator-prey model with modified Leslie-Gower and Holling-type II scheme, Nonlinear Analysis: Real World Applications Vol.9, (2008) 20552067.

[36] R. Yafia, F. El Adnani and H. Talibi, Stability of limit cycle in a predator-prey model with modified Leslie-Gower and Hollingtype II schemes with time delay. Applied Mathematical Sciences, Vol. 1, no. 3, (2007) pp 119 - 131.

[37] E. Beretta, Y. Kuang, Global analyses in some delayed ratio-depended predator-prey systems, Nonlinear Anal. Theory Methods Appl. 32 (3) (1998) 381-408.

[38] A.F. Nindjin, M.A. Aziz-Alaoui and M. Cadivel, Analysis of a Predator-Prey Model with Modified Leslie-Gower and HollingType II Schemes with Time Delay, Nonlinear Anal. Real World Appl., 7(5), (2006) 1104-1118. Theoretical Biology 245 (2007) 220-229.

[39] Sze-Bi Hsu and Tzy-Wei Hwang, Hopf bifurcation for a predator-prey system of Holling and Leslie type, Taywanese journal of Mathematics Vol. 3, No. 1, pp. 35-53, March 1999.

[40] S. Chakraborty, S. Pal, N. Bairagi, Predator-prey interaction with harvesting: mathematical study with biological ramifications, Applied Mathematical Modelling 36 (2012) 4044-4059.

[41] C.W. Clark, Mathematical Bioeconomics: The Optimal Management of Renewable Resources, second ed., John Wiley and Sons, New York, 1990.

[42] B. Roy and S. K. Roy, Analysis of prey-predator three species models with vertebral and invertebral predators, International Journal Dynamics and Control 3 (3), (2015) 306-312.

[43] S. K. Roy and B. Roy, Analysis of prey-predator three species fishery model with harvesting including prey refuge and $\mathrm{mi}-$ gration, International Journal of Bifurcation and Chaos 26 (02), (2016) 1650022.

[44] B. Roy, S. K. Roy and D. B. Gurung, Holling-Tanner model with Beddington-DeAngelis functional response and time delay introducing harvesting, Mathematics and Computers in Simulation 142 (2017) 1-14.

[45] B. Roy, S. K. Roy and M. H. A. Biswas, Effects on prey-predator with different functional response, International Journal of Biomathematics 10 (08), (2017) 1750113.

[46] Birkoff G. and Rota G.C., Ordinary Differential Equations. Ginn; (1982). 